\title{
Réponse à la sécheresse de conifères indigènes et exotiques: une étude dendroécologique
}

Mathieu Lévesque Andreas Rigling

Peter Brang
Institut fédéral de recherches sur la forêt, la neige et le paysage et Lamont-Doherty Earth Observatory (USA)* Institut fédéral de recherches sur la forêt, la neige et le paysage $(\mathrm{CH})$

Institut fédéral de recherches sur la forêt, la neige et le paysage $(\mathrm{CH})$

\section{Réponse à la sécheresse de conifères indigènes et exotiques: une étude dendroécologique}

\begin{abstract}
La sensibilité à la sécheresse de cinq conifères a été étudiée le long d'un gradient climatique couvrant le versant nord des Alpes suisses, le Plateau suisse, les contreforts du Jura et les Alpes centrales. Trois espèces indigènes, l'épicéa, le pin sylvestre et le mélèze, et deux espèces non indigènes, le sapin de Douglas et le pin noir, ont été analysées. Au total, 770 arbres sur 14 sites ont été échantillonnés. Pour évaluer la sensibilité des conifères à la sécheresse, des analyses dendrochronologiques ont été faites sur la période 1941-2006. Sur les sites secs dans les Alpes centrales, les déficits hydriques printaniers réduisent fortement la croissance, alors que sur les sites mésophiles situés sur le Plateau suisse et les contreforts du Jura, les sécheresses estivales freinent significativement la croissance. L'épicéa, le mélèze et le pin sylvestre sont, selon les stations, les espèces les plus vulnérables aux sécheresses extrêmes, ce qui implique que leur performance à long terme et leur survie peuvent être compromises si le climat devient plus chaud et plus sec. A l'inverse, la croissance du douglas et du pin noir montre une sensibilité moindre à la sécheresse et une croissance supérieure que celle des espèces indigènes le long du gradient climatique. Sélectionner des espèces ayant des stratégies différentes, mais complémentaires vis-à-vis de la sécheresse pourrait sécuriser la productivité et l'efficience d'utilisation de l'eau des peuplements forestiers et ainsi augmenter leur résistance au changement climatique.
\end{abstract}

Keywords: drought response, growth, Pinus sylvestris, Picea abies, Larix decidua, Pinus nigra, Pseudotsuga menziesii, Switzerland, Italy

doi: $10.3188 /$ szf.2015.0372

* Columbia University, Palisades/NY 10964 (USA), courriel mathieu.levesque@wsl.ch

L es plus récentes projections climatiques indiquent une augmentation des températures de 2.7 à $4.1^{\circ} \mathrm{C}$ selon le scénario d'émission moyen $\mathrm{A} 1 \mathrm{~B}$ et une réduction marquée des précipitations en période estivale d'ici la fin du siècle en Suisse. La fréquence, la durée et la sévérité des sécheresses extrêmes vont aussi augmenter (C2SM 2011). Un climat plus chaud et plus sec et des sécheresses exceptionnelles peuvent réduire considérablement la croissance et la vitalité des peuplements forestiers (Bréda et al 2006). Par exemple, les sécheresses exceptionnelles de 1976 et 2003 sont à l'origine du dépérissement du pin sylvestre (Pinus sylvestris L.) (Bigler et al 2006) et de l'épicéa (Picea abies [L.] Karst; Becker et al 1995). Avec les changements climatiques en cours, il devient donc urgent de mieux comprendre la réponse à la sécheresse et au climat des essences forestières afin d'élaborer des mesures adaptatives de gestion forestière.

Les études dendroécologiques permettent d'analyser rétrospectivement la réponse au climat des arbres. Ce genre d'étude a aussi l'avantage de permettre l'échantillonnage sur de larges gradients climatiques et édaphiques (e.g. Lebourgeois et al 2010). Des études dendrochronologiques ont démontré des réductions en croissance à la suite de sécheresses pour l'épicéa en France (Desplanque et al 1999, Lebourgeois et al 2010) et en Allemagne (Zang 2011, Zang et al 2012, van der Maaten-Theunissen et al 2013). En Suisse, notamment en Valais, des réductions importantes de la croissance et même des épisodes de mortalité ont été observés pour le pin sylvestre à la suite des sécheresses exceptionnelles des années 2000 (Bigler et al 2006). La plupart des études antérieures sont basées sur la mesure de la largeur des cernes annuels. Conséquemment, elles ne donnent aucune indication directe dans les processus écophysiologiques sous-jacents. Cette limitation peut être surmontée en utilisant en complémentarité la composition isotopique ( $\mathrm{C}^{13}$ et $\left.\mathrm{O}^{18}\right)$ des cernes annuels.

L'isotope stable du carbone $\left({ }^{13} \mathrm{C}\right)$ est directement lié à l'absorption de $\mathrm{CO}_{2}$ et l'efficience intrin- 
sèque d'utilisation de l'eau, à savoir le rapport entre le taux d'assimilation et la conductance stomatique lors de la photosynthèse (McCarroll \& Loader 2004). Ainsi, ${ }^{13} \mathrm{C}$ dans les cernes des arbres indique le degré de stress hydrique lors de sécheresse. L'isotope stable de l'oxygène $\left({ }^{18} \mathrm{O}\right)$ fournit des informations sur la source d'eau utilisée par l'arbre, la conductance stomatique et la transpiration foliaire (McCarroll \& Loader 2004).

L'objectif principal de cette étude est de déterminer la vulnérabilité à la sécheresse de trois espèces indigènes, l'épicéa, le pin sylvestre et le mélèze ( $L a$ rix decidua Mill.), contrairement à deux espèces non indigènes, le sapin de Douglas (Pseudotsuga menziesii var. menziesii [Mirb.] Franco) et le pin noir (Pinus nigra Arn.), le long d'un large gradient climatique en Suisse (figures 1 et 2 ). A cette fin, l'analyse simultanée de la croissance radiale et des variations isotopiques dans les cernes annuels est utilisée. Les résul- tats présentés résument une thèse (Lévesque 2013) et trois articles scientifiques (Lévesque et al 2013, Lévesque et al 2014a, Lévesque et al 2014b).

\section{Matériel et méthodes}

\section{Zone d'étude}

La zone d'étude se trouve en Suisse et au nord de l'Italie et comprend quatre régions biogéographiques: le nord des Alpes, le Plateau suisse, les contreforts du Jura et les vallées alpines sèches des Alpes centrales (tableau 1, figure 1). L'ordre des régions géographiques correspond à un gradient climatique où les précipitations annuelles diminuent et la fréquence des sécheresses estivales augmente. Dans la zone d'étude, nous avons sélectionné 14 peuplements qui répondaient à trois critères: 1) la présence d'au moins une espèce non indigène: le douglas ou le pin noir,

\begin{tabular}{|c|c|c|c|c|c|c|c|c|c|c|c|c|}
\hline Site & \begin{tabular}{c|} 
Code \\
du \\
site
\end{tabular} & Région & Espèces & $X(m)$ & $Y(m)$ & $\begin{array}{l}\text { Altitude } \\
(\mathrm{m})\end{array}$ & $\begin{array}{l}\text { Exposi- } \\
\text { tion }\end{array}$ & $\begin{array}{c}\text { Pente } \\
(\%)\end{array}$ & $\begin{array}{l}\text { Réserve } \\
\text { utile en eau } \\
\text { du sol }(\mathrm{mm})\end{array}$ & $\begin{array}{c}\text { Température } \\
\text { annuelle } \\
\text { moyenne }\left({ }^{\circ} \mathrm{C}\right)\end{array}$ & $\begin{array}{l}\text { Précipitation } \\
\text { annuelle } \\
\text { moyenne }(\mathrm{mm})\end{array}$ & $\begin{array}{c}\text { Bilan } \\
\text { hydrique cli- } \\
\text { matique }(\mathrm{mm})\end{array}$ \\
\hline Schlanders & SC & $\begin{array}{c}\text { Alpes } \\
\text { centrales }\end{array}$ & pn, mé, ps & 857100 & 170300 & 1145 & so & 45 & 128 & 7.0 & 493 & -53 \\
\hline Aoste & $\mathrm{AO}$ & $\begin{array}{c}\text { Alpes } \\
\text { centrales }\end{array}$ & $\begin{array}{l}\text { pn, do, } \\
\text { mé, ps, ép }\end{array}$ & 607200 & 68200 & 1150 & so & 75 & 82 & 7.8 & 691 & -43 \\
\hline $\begin{array}{l}\text { Goppen- } \\
\text { stein }\end{array}$ & $\mathrm{GO}$ & $\begin{array}{c}\text { Alpes } \\
\text { centrales }\end{array}$ & do, mé, ép & 624750 & 134250 & 1225 & NO & 70 & 84 & 6.3 & 931 & -19 \\
\hline Crans & $C R$ & $\begin{array}{c}\text { Alpes } \\
\text { centrales }\end{array}$ & pn, mé, ép & 601150 & 126400 & 1260 & so & 27 & 186 & 6.0 & 954 & -15 \\
\hline Steckborn & ST & $\begin{array}{l}\text { Plateau } \\
\text { suisse }\end{array}$ & pn, mé, ps & 716950 & 280000 & 535 & $\mathrm{O}$ & 20 & 82 & 8.7 & 932 & -11 \\
\hline $\begin{array}{l}\text { Yverdon- } \\
\text { les-Bains }\end{array}$ & YB & Jura & $\begin{array}{l}\text { pn, do, } \\
\text { mé, ps, ép }\end{array}$ & 547300 & 191250 & 570 & SE & 15 & 94 & 9.1 & 1071 & -10 \\
\hline Bienne & $\mathrm{BI}$ & Jura & $\begin{array}{l}\text { pn, do, } \\
\text { mé, ps, ép }\end{array}$ & 587300 & 223800 & 750 & $S$ & 35 & 119 & 8.0 & 1240 & 12 \\
\hline Neuhaus & NE & $\begin{array}{c}\text { Nord } \\
\text { des } \\
\text { Alpes }\end{array}$ & $\begin{array}{l}\text { pn, mé, } \\
\text { ps, ép }\end{array}$ & 628400 & 170300 & 620 & so & 61 & 59 & 8.7 & 1173 & 15 \\
\hline Merligen & ME & $\begin{array}{c}\text { Nord } \\
\text { des } \\
\text { Alpes }\end{array}$ & pn, ps, ép & 622700 & 172700 & 750 & NO & 62 & 68 & 8.6 & 1170 & 19 \\
\hline Sargans & SA & $\begin{array}{l}\text { Nord } \\
\text { des } \\
\text { Alpes }\end{array}$ & do, mé, ps & 754150 & 216475 & 700 & $S$ & 47 & 159 & 8.2 & 1275 & 30 \\
\hline
\end{tabular}

Tab. 1 Caractéristiques des sites étudiés. Les sites sont classés selon le bilan hydrique climatique (du plus sec au plus humide). Espèces: pn: pin noir; do: douglas; mé: mélèze; ps: pin sylvestre; ép: épicéa. Le bilan hydrique climatique est défini comme la somme des précipitations moins la somme de l'évapotranspiration potentielle des mois d'avril à septembre. L'évapotranspiration potentielle est estimée selon la méthode de Thornthwaite (1948). Le bilan hydrique climatique est positif lorsque les précipitations excèdent l'évapotranspiration potentielle et négatif dans le cas d'un déficit en eau. Adapté de Lévesque et al (2014a) 


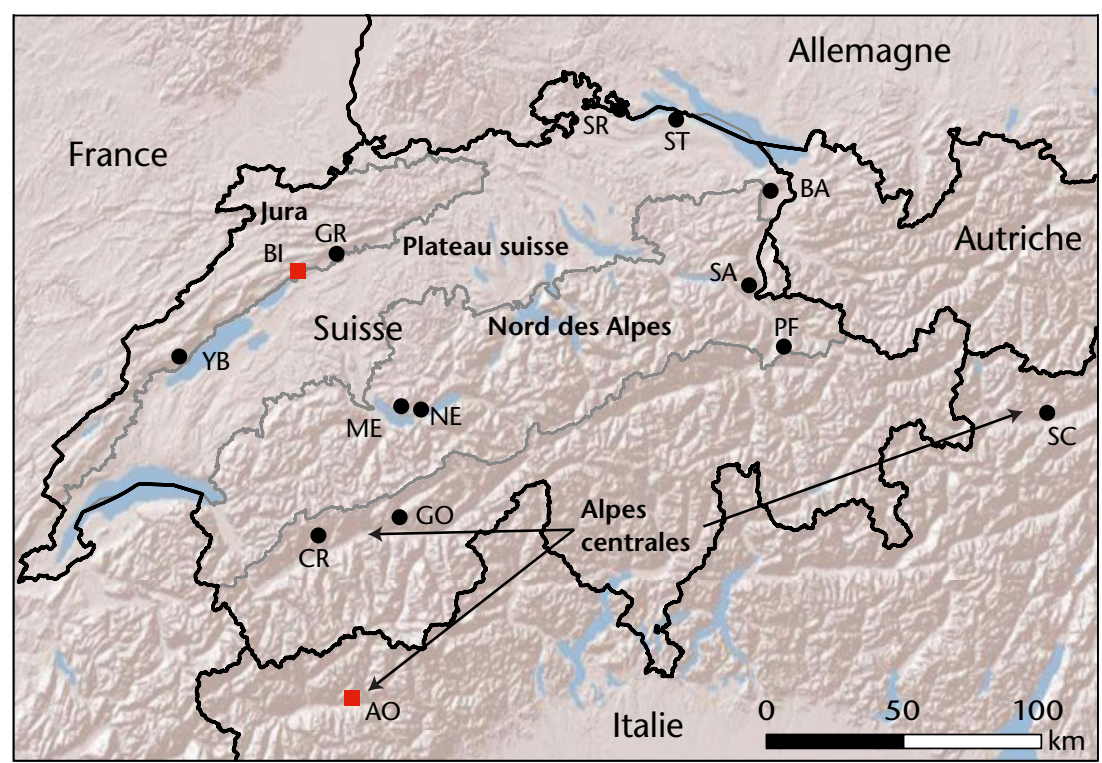

Fig. 1 Sites étudiés. Les sites utilisés exclusivement pour l'analyse de la croissance radiale sont indiqués par les points noirs, tandis que les sites ayant servi à l'analyse combinée des isotopes stables et de la croissance radiale sont indiqués par des carrés rouges. Voir tableau 1 pour le code des sites. La carte de base a été reproduite avec la permission de swisstopo (JA100118)

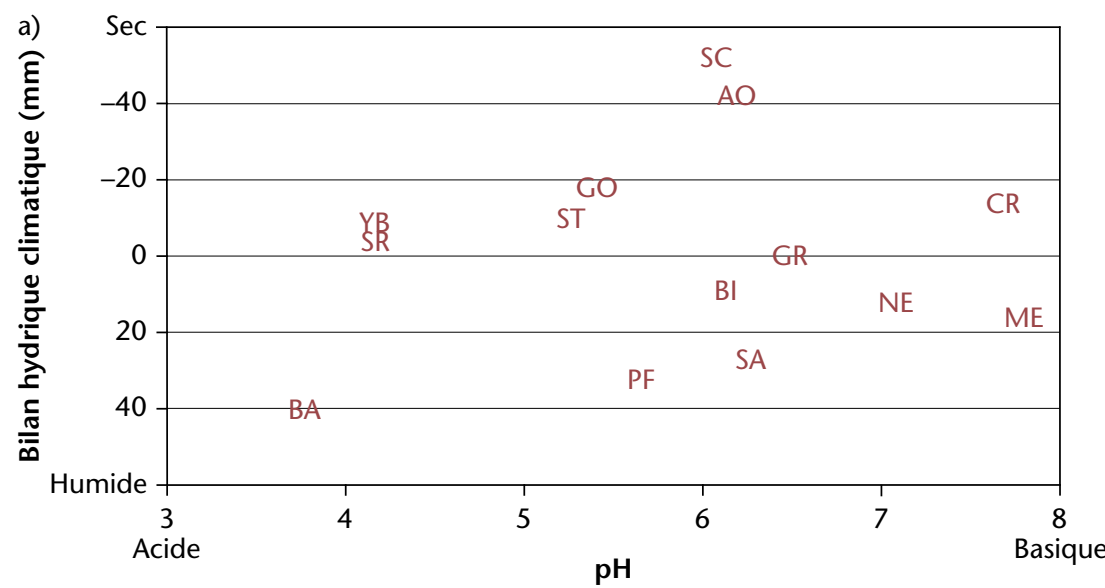

b)

\begin{tabular}{|l|c|c|c|}
\hline \multirow{2}{*}{ Espèce } & \multirow{2}{*}{$\begin{array}{c}\text { Nombre } \\
\text { de sites }\end{array}$} & \multicolumn{2}{|c|}{ Gradient échantillonné } \\
\cline { 3 - 4 } & & $\mathrm{pH}$ & Bilan hydrique climatique $(\mathrm{mm})$ \\
\hline Epicéa & 11 & 3.7 à 7.7 & -43 à 43 \\
\hline Pin sylvestre & 11 & 3.7 à 7.7 & -53 à 43 \\
\hline Mélèze & 13 & 3.7 à 7.6 & -53 à 43 \\
\hline Douglas & 8 & 3.7 à 6.2 & -43 à 43 \\
\hline Pin noir & 10 & 3.7 à 7.7 & -53 à 43 \\
\hline
\end{tabular}

Fig. 2 Ecogramme des sites étudiés selon l'acidité des sols et le bilan hydrique climatique en a). Gradient d'acidité et d'humidité des sites échantillonnés en fonction des espèces en b). Voir le tableau 1 pour le code des sites et la liste des espèces présentes à chaque site.

2) l'occurrence d'au moins trois des cinq espèces étudiées, 3) la présence de peuplements équiens où l'âge minimal des arbres est de 70 ans. A chaque site, un profil de sol a été creusé pour déterminer le type de sol, son $\mathrm{pH}$ et sa capacité de rétention en eau (AG Bodenkunde 2005, Walthert et al 2013). Les 14 sites étudiés couvrent une large gamme de conditions pédoclimatiques allant des stations sèches, acides et pauvres aux stations mésophiles, basiques et riches (figure 2). Toutes les espèces étaient présentes le long du gradient échantillonné (tableau 1, figure 2).

\section{Mesures dendrochronologiques}

A chaque site, entre 11 et 15 arbres dominants par espèce ont été échantillonnés en prenant deux carottes par arbre à l'aide d'une tarière de Pressler. Les carottes ont été montées sur des supports en bois, et la largeur de chaque cerne annuel a été mesurée avec un microscope relié à une table de positionnement numérique et le logiciel LINTAB TSAP (Rinntech, Heidelberg, Allemagne). Les séries de cernes individuels ont été ensuite interdatées visuellement et vérifiées statistiquement à l'aide du logiciel COFECHA (Holmes 1983). Les chronologies individuelles ont été standardisées à l'aide de courbe de lissage cubique (polynôme d'ordre 3) sur des périodes de 32 ans. Cette technique permet d'éliminer les signaux non liés au climat, notamment les fluctuations de croissance dues à l'âge de l'arbre, et d'uniformiser les séries individuelles. Finalement, les chronologies standardisées ont été moyennées par essence et par site. La sensibilité moyenne qui quantifie la variabilité interannuelle dans la croissance radiale a été calculée sur les données brutes de même que l'autocorrélation d'ordre 1 qui mesure l'influence des conditions de l'année précédente sur la croissance de l'année courante (Fritts 1976).

\section{Données climatiques}

Pour les sites suisses, les données climatiques journalières ont été interpolées à l'aide du modèle DAYMET (Thornton et al 1997) pour la période 1941-2006 à partir de plusieurs stations de MétéoSuisse situées à proximité de chaque site. Pour les sites italiens, les données climatiques ont été obtenues à partir de la station de mesure la plus proche de la zone d'étude $(5 \mathrm{~km}$ pour le site Aoste, $3 \mathrm{~km}$ pour le site Schlanders). Pour tester l'effet de la disponibilité en eau du sol sur la croissance des arbres, l'indice de sécheresse de Palmer, qui est une mesure de l'humidité du sol, a été calculé pour chaque site (Wells et al 2004) en utilisant la température moyenne mensuelle, la somme mensuelle des précipitations, la capacité de rétention en eau du sol et la latitude (figure 3).

\section{Analyses}

Les réponses de croissance et d'échanges gazeux des arbres au bilan hydrique sur site sec (Aoste) et sur site mésophile (Bienne) ont été évaluées avec des corrélations (Lévesque et al 2013). Les coefficients de corrélation mensuels ont été calculés pour l'année précédente (de juin à décembre) et l'année de formation du cerne de croissance (janvier à septembre) pour la période 1960-2009, afin de déterminer les mois de l'année où la disponibilité en eau du sol est la plus critique pour la croissance, la photosynthèse et la conductance stomatique des conifères.

Pour déterminer les réactions de croissance des espèces aux sécheresses extrêmes le long du gradient climatique échantillonné, l'analyse par superposition 


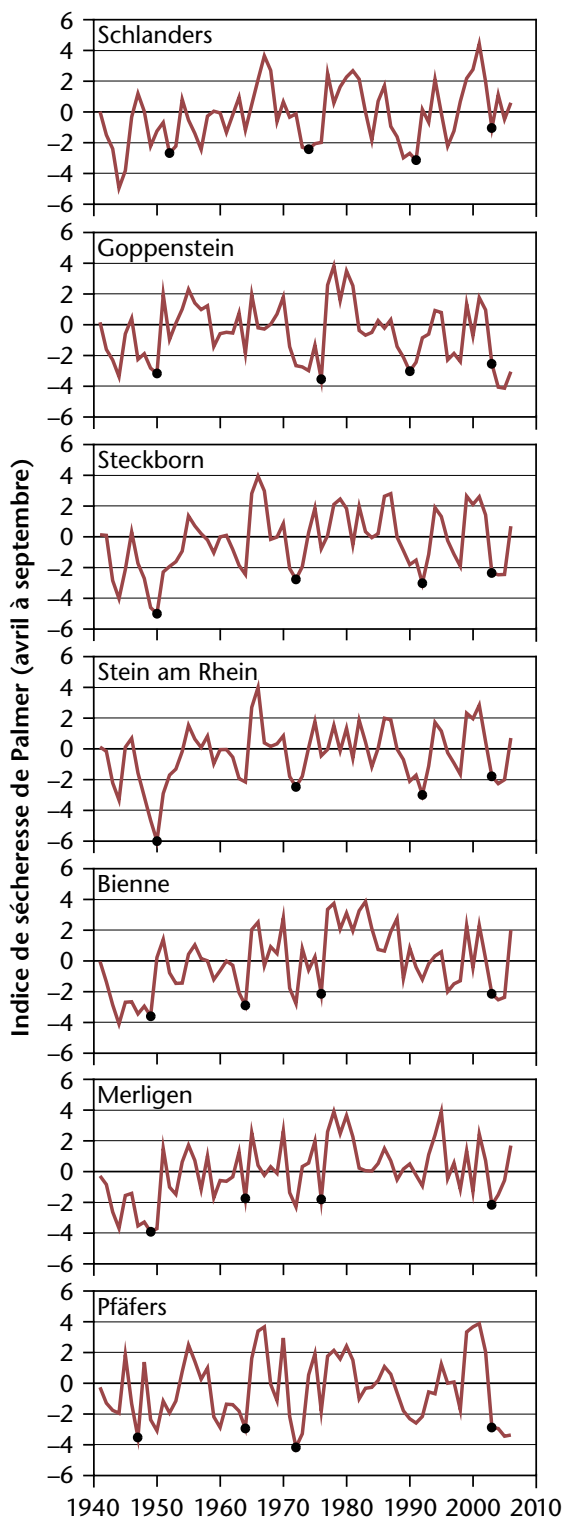

Année
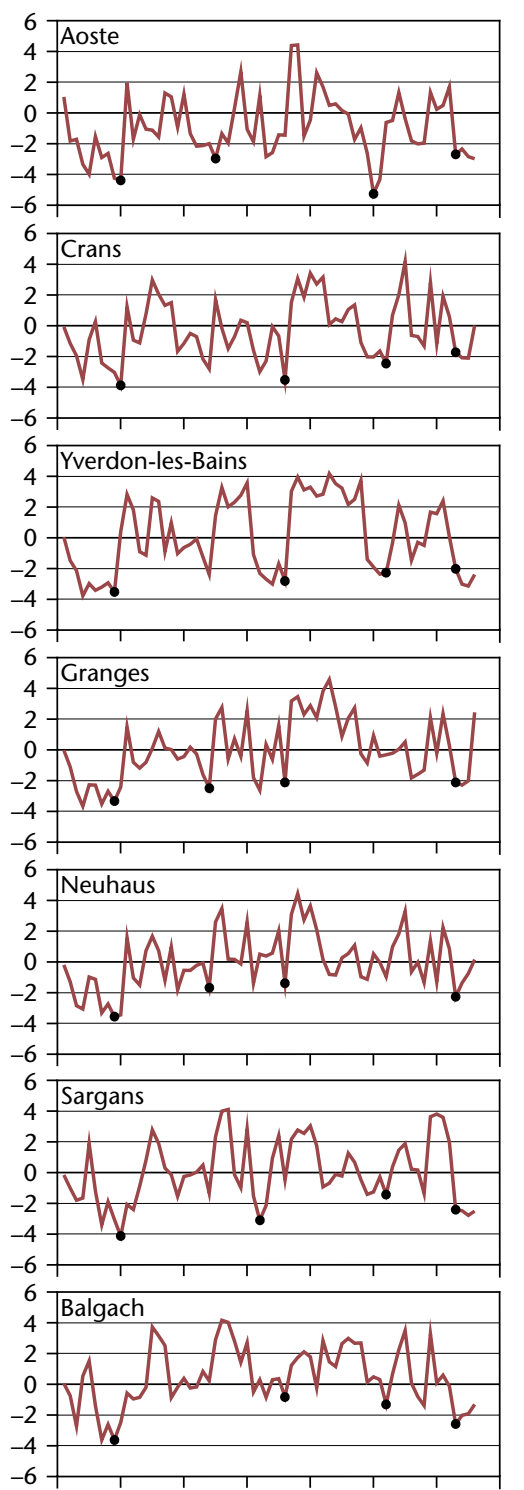

19401950196019701980199020002010 Année

Fig. 3 Indice de sécheresse de Palmer d'avril à septembre des sites étudiés pour la période 1941-2006. L'indice de sécheresse varie habituellement entre -4 (conditions extrêmement sèches) et +4 (conditions extrêmement humides), tandis que des valeurs près de 0 indiquent des conditions d'humidité moyennes. Les points noirs indiquent les années de sécheresse exceptionnelles utilisées dans l'analyse par superposition des années de sécheresse extrême (figure 4). Adaptée de Lévesque et al (2014a). Autorisation de reproduction 3614920372878.

\begin{tabular}{|l|c|c|c|c|c|c|}
\hline Espèce & $\begin{array}{c}\text { Nombre } \\
\text { d'arbres }\end{array}$ & Age & $\begin{array}{c}\text { Diamètre } \\
(\mathrm{cm})\end{array}$ & $\begin{array}{c}\text { Hauteur } \\
(\mathrm{m})\end{array}$ & $\begin{array}{c}\text { Largeur de } \\
\text { cerne }(\mathrm{mm})\end{array}$ & SM \\
\hline Epicéa & 156 & $103(22)$ & $47.8(9.2)$ & $30.2(6.0)$ & $2.39(0.83)$ & 0.23 \\
\hline Pin sylvestre & 163 & $109(23)$ & $43.3(7.8)$ & $25.5(6.2)$ & $1.38(0.65)$ & 0.23 \\
\hline Mélèze & 187 & $99(18)$ & $46.1(9.7)$ & $28.6(6.0)$ & $2.00(0.66)$ & 0.29 \\
\hline Douglas & 115 & $93(9)$ & $70.2(14.1)$ & $36.4(7.8)$ & $3.40(0.95)$ & 0.19 \\
\hline Pin noir & 149 & $109(19)$ & $49.2(8.6)$ & $26.2(5.4)$ & $1.68(0.44)$ & 0.21 \\
\hline
\end{tabular}

Tab. 2 Caractéristiques moyennes et erreurs types (entre parenthèses) des espèces étudiées. $S M=$ sensibilité moyenne.

des années de sécheresse extrême a été utilisée (Orwig \& Abrams 1997). L'analyse par superposition est un test statistique non paramétrique de randomisation permettant d'évaluer les écarts significatifs de croissance avant, pendant et après les années de sécheresse. A chaque site, les trois années non

consécutives les plus sèches et l'année extrême 2003 ont été analysées (figure 2).

\section{Résultats}

\section{Caractéristiques des arbres échantillonnés et séries dendrochronologiques}

Le diamètre moyen à hauteur de poitrine des arbres échantillonnés variait de 24.7 à $84.4 \mathrm{~cm}$ et leur hauteur de 9.5 à $43.3 \mathrm{~m}$. La croissance radiale moyenne variait de 0.55 à $5.25 \mathrm{~mm}$ par an, avec la plus faible croissance enregistrée pour le pin sylvestre et la plus élevée pour le douglas. La sensibilité moyenne, qui mesure la variabilité interannuelle de la croissance et la sensibilité de celle-ci au climat, variait entre 0.15 et 0.64 , et était plus élevée sur les sites secs que sur les sites humides. La sensibilité la plus élevée a été enregistrée pour le mélèze (moyenne 0.29 ), suivi par l'épicéa et le pin sylvestre (moyenne 0.23; tableau 2).

\section{Réponse spécifique des espèces aux années de sécheresse extrême}

Lors des années extrêmement sèches, le mélèze et l'épicéa ont montré les plus fortes réductions de croissance (figure 4). La croissance de ces deux espèces a fortement diminué (jusqu'à environ $-50 \%$ ) au cours des années de sécheresse extrême sur les sites les plus secs (vallées alpines sèches, sites: SC, $\mathrm{AO}, \mathrm{GO}$ et $\mathrm{CR}$ ). De plus, l'épicéa et le mélèze ont montré le plus grand nombre de réductions significatives de croissance lors des sécheresses extrêmes. Le douglas n'a pas été significativement influencé par la sécheresse, sauf à deux sites secs (AO et GO) dans les Alpes centrales. De même, la croissance du pin noir et du pin sylvestre a été moins affectée lors de sécheresses sévères, et ils ont seulement montré des réductions significatives de croissance à quelques sites. Toutes les espèces ont montré des réductions significatives de croissance pendant les épisodes de sécheresse extrême sur les sites les plus secs, mais la croissance de la plupart des espèces est revenue à son niveau d'avant la sécheresse un ou deux ans après.

\section{Réponses physiologiques et de la croissance des conifères au déficit hydrique}

La disponibilité en eau l'automne, l'hiver et au printemps influençait significativement les échanges gazeux et la croissance radiale des cinq espèces sur site sec (Aoste), tandis que sur site mésophile (Bienne) les mêmes espèces étaient essentiellement sensibles au bilan hydrique estival (tableau 3). Sur site sec, l'isotope ${ }^{13} \mathrm{C}$, qui sert d'indice d'activité photosynthétique et de transpiration au niveau foliaire, était significativement et négativement corrélé au bilan hydrique printanier et estival, ce qui indique une réduction de la photosynthèse et de la transpiration foliaire durant des printemps et étés secs. L'isotope 


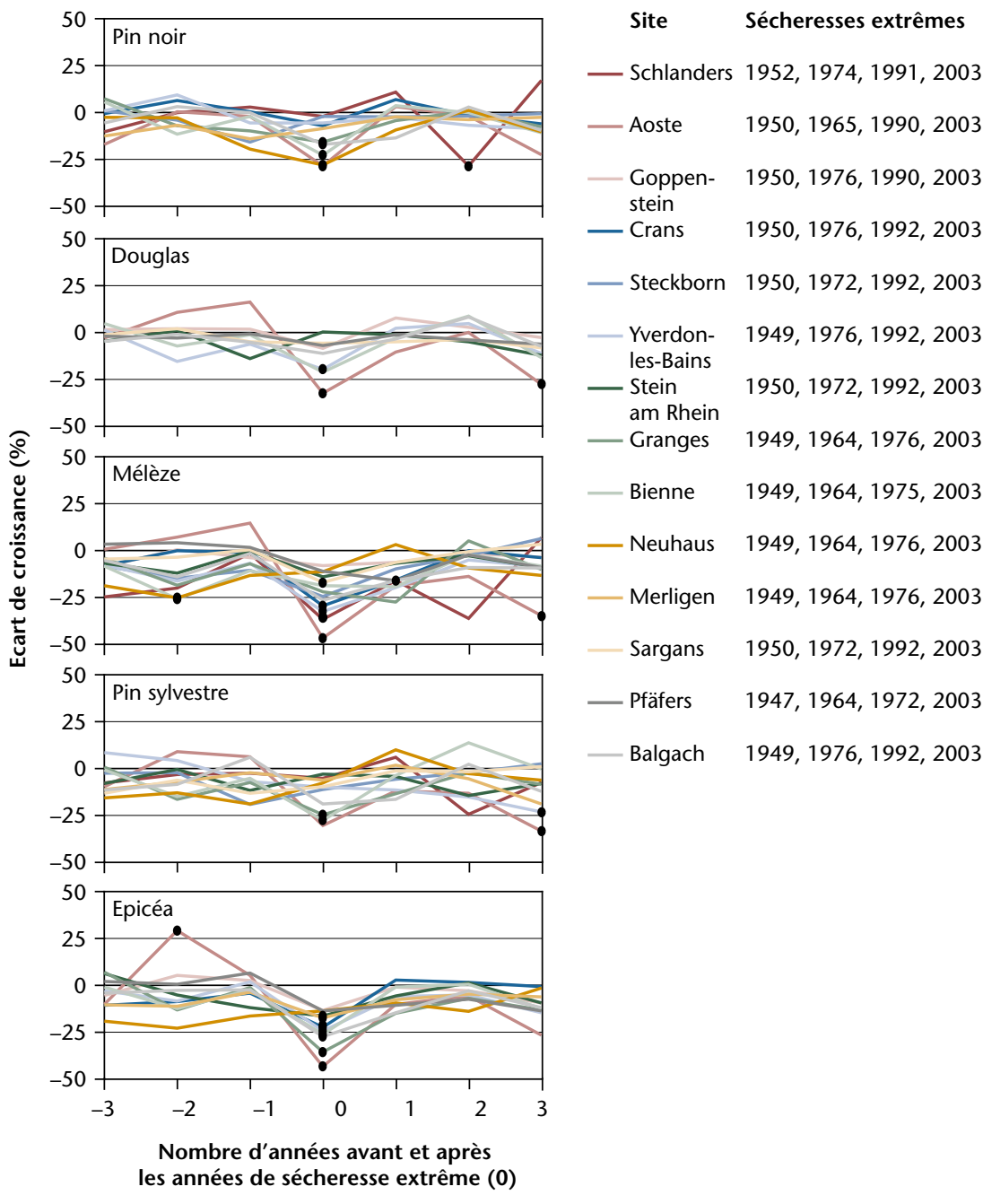

Fig. 4 Analyse par superposition des années de sécheresse extrême montrant les écarts de croissance avant, pendant (année 0) et après les années de sécheresse extrême identifiées à chaque site d'étude. Pour chaque site, les trois années non consécutives les plus sèches et l'année extrême 2003 ont été sélectionnées à partir des séries temporelles de l'indice de sécheresse de Palmer (figure 3). Les points noirs sur les courbes indiquent des écarts de croissance significatifs $(P<0.05)$. Adaptée de Lévesque et al (2014a). Autorisation de reproduction 3614920372878

\section{Discussion}

\section{Réponse de la croissance en fonction de l'aridité des sites}

Nos résultats mettent en évidence une grande variabilité de la réponse de la croissance des arbres à la sécheresse selon l'aridité des sites. Les arbres situés dans les Alpes centrales (conditions sèches) montrent des taux de croissance inférieure et une sensibilité supérieure aux fluctuations climatiques que les arbres poussant sur les sites mésophiles (Plateau suisse, Jura) et humides (nord des Alpes). Sur les sites secs situés dans les Alpes centrales (p. ex. site Aoste, tableau 3), la disponibilité en eau en été influence peu la croissance radiale, tandis que des conditions sèches en hiver et au printemps entravent fortement la croissance de toutes les espèces. Les sécheresses printanières peuvent fortement réduire la croissance des arbres dans les vallées sèches des Alpes centrales puisque c'est durant le printemps que la majeure partie de la croissance se produit (Eilmann et al 2011). Pour faire face aux étés secs des vallées intérieures alpines, les arbres concentrent leur croissance radiale au printemps (Eilmann et al 2011).

Contrairement aux réponses trouvées sur les sites secs dans les Alpes, les conifères échantillonnés sur les sites mésophiles (Plateau suisse, Jura) sont principalement sensibles aux sécheresses estivales (p. ex. site Bienne, tableau 3). Sur site mésophile, la croissance peut se produire pendant toute la saison de croissance aussi longtemps que les déficits hydriques ne sont pas assez sévères pour provoquer la fermeture des stomates et une réduction de l'activité photosynthétique (Lévesque et al 2013). Lorsque les conditions deviennent trop sèches, les arbres réduisent leur conductance stomatique et donc la transpiration foliaire et la photosynthèse, entraînant une réduction de la croissance en période de sécheresse estivale (Lévesque et al 2013). Nos résultats sont en ligne avec la forte sensibilité à la sécheresse estivale de diverses espèces de conifères rapportée pour les forêts mésophiles en Espagne (Pasho et al 2011) et en France (Lebourgeois et al 2010). Globalement, la forte relation entre la disponibilité en eau en été, la transpiration, la photosynthèse et la croissance sur les sites mésophiles suggère que les conifères sur ces sites peuvent aussi être vulnérables aux sécheresses estivales sévères, surtout $s i$ la longueur et l'intensité de celles-ci augmentent comme le suggèrent les scénarios climatiques (C2SM 2011).

\section{Réponses de la croissance des espèces à la sécheresse}

Le long du gradient climatique étudié, l'épicéa et le mélèze sont les espèces les plus vulnérables à la sécheresse estivale. Ces deux espèces ont montré les plus fortes réductions de croissance lors d'années extrêmement sèches, et cela particulièrement sur les sites les plus secs situés dans les vallées alpines sèches du 


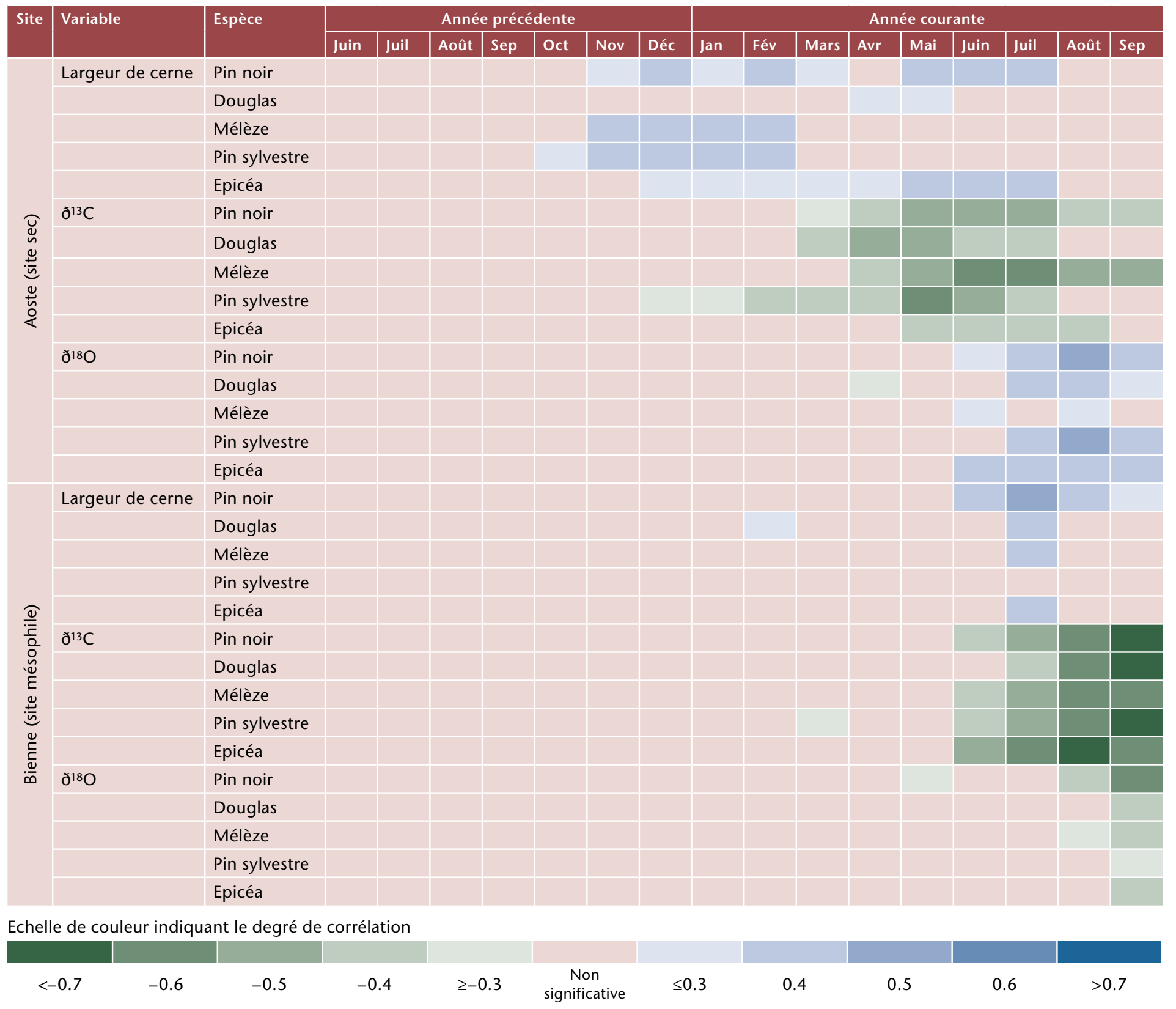

Tab. 3 Corrélations entre les variables largeur de cerne, $\partial^{13} \mathrm{C}$ et ${ }^{18} \mathrm{O}$ contenus dans les cernes annuels et le bilan hydrique pour cinq espèces sur site sec (Aoste) et sur site mésophile (Bienne). Les mois de l'année précédente et courante présentant des corrélations significatives $(P<0.05)$ sont indiqués en couleurs. Adapté de Lévesque et al (2013).

Valais (sites GO et CR), d'Aoste (site AO) et du Vinschgau (site SC). Les sécheresses extrêmes comme celle de 2003 peuvent réduire la croissance et la vigueur de l'épicéa, du mélèze et du pin sylvestre pendant plus de trois ans (figure 4). Une période de récupération prolongée et une vigueur réduite augmentent la susceptibilité de ces espèces à être attaquées par les insectes ravageurs et les agents pathogènes. Ainsi, la performance à long terme ou même la survie de l'épicéa qui possède un système racinaire moins profond que d'autres espèces (Schmid \& Kazda 2002, Zang 2011) et qui est très sensible aux cavitations (Hentschel et al 2014) et aux attaques de scolytes (Wermelinger 2004), de même que le mélèze, une espèce ayant un contrôle stomatique inefficace (Leo et al 2014), peuvent être compromises sous un climat plus sec.

En comparaison avec le sapin de Douglas et le pin noir, la croissance du pin sylvestre montre éga- lement une sensibilité plus élevée (0.23; tableau 2) aux variations climatiques (Lévesque et al 2014a). Lors de déficits hydriques, cette espèce réduit fortement ses échanges gazeux afin de réduire le risque de défaillance hydraulique, mais ceci limite simultanément sa photosynthèse et sa croissance (Eilmann et al 2010). Ultimement, des épisodes de sécheresse exceptionnelle peuvent réduire la vigueur et provoquer la mortalité du pin sylvestre (Bigler et al 2006). Nos résultats et ceux d'études précédentes (Bigler et al 2006, Weber et al 2007) confirment que la résistance de cette espèce à la sécheresse est limitée, et ce particulièrement sur sites secs.

Contrairement aux espèces indigènes, la croissance du pin noir et du douglas est moins affectée lors de sécheresses. Les deux espèces ont été en mesure de maintenir des niveaux de croissance élevés et ont montré des valeurs de sensibilité moyenne plus 
faibles que celles calculées pour l'épicéa, le mélèze et le pin sylvestre (tableau 2). Ces deux espèces possèdent un contrôle stomatique efficace pour réduire la perte d'eau par transpiration et sont capables de maintenir un système hydraulique fonctionnel lors de sécheresses (Lassoie \& Salo 1981, Lebourgeois et al 1998). Elles ont aussi une grande capacité à vite compenser une baisse de croissance due à la sécheresse (Eilmann \& Rigling 2012, Lévesque et al 2014a). Bien que certaines études suggèrent que le douglas peut être vulnérable aux sécheresses extrêmes au stade jeune (Jansen et al 2013, Sergent et al 2014), nos résultats indiquent qu'au stade mature cette espèce est peu affectée par les sécheresses estivales en Suisse. De plus, cette espèce est la plus productive le long du gradient climatique échantillonné, et ce même sur les sites les plus secs dans les Alpes centrales. Ces caractéristiques font du douglas et du pin noir des espèces alternatives intéressantes à l'épicéa et au pin sylvestre.

\section{Conclusions et pertinence}

Nous avons évalué la sensibilité à la sécheresse de cinq conifères indigènes et exotiques le long d'un gradient pédoclimatique couvrant quatre régions biogéographiques en Suisse. Sur les sites secs situés dans les Alpes centrales, la disponibilité en eau avant et au début de la période de végétation est essentielle pour la croissance des arbres, alors que sur les sites mésophiles sur le Plateau suisse et au pied du Jura, la disponibilité en eau en été est critique. Les sécheresses estivales entravent de manière significative la croissance de l'épicéa et du mélèze le long du gradient climatique. Toutefois, sur les stations humides, le mélèze montre une résistance à la sécheresse supérieure à celle de l'épicéa (figure 4). Dans l'ensemble, nos résultats suggèrent que la performance à long terme ou même la survie de l'épicéa et du mélèze sur des sites secs risquent d'être compromises si le climat devient plus chaud et sec. Au contraire, le douglas (var. menziesii) montre une sensitivité moindre à la sécheresse et une croissance supérieure sur stations modérément sèches à humides. Même si le pin noir est peu apprécié des forestiers à cause de ses mauvaises qualités technologiques et de ses débouchés limités, cette essence a été plantée, et avec succès, pendant une certaine période en Suisse. Nos résultats indiquent que la croissance de cette espèce est moins sensible à la sècheresse que le pin sylvestre sur site secs. Toutefois, les sécheresses extrêmes peuvent augmenter la sensibilité du pin noir à l'agent pathogène Sphaeropsis sapinea comme en témoignent les signes de dépérissements observés sur les contreforts du Jura en 2015. Ainsi, le douglas pourrait être utilisé comme essence de remplacement ou mélangé aux espèces plus sensibles à la sécheresse (l'épicéa et le pin sylvestre) pour accroître la résistance des peuplements aux futures conditions climatiques. Cela permettrait de maintenir des espèces à haute valeur économique tout en garantissant d'autres services écosystémiques (p. ex. forêts de protection, filtration de l'eau et de l'air).

Soumis: 27 janvier 2015, accepté (avec comité de lecture): 4 juin 2015

\section{Remerciement}

Nous remercions A. Bürgi, G. Vacchiano et les forestiers suisses et italiens pour leur aide apportée lors de la sélection des sites et les autorisations délivrées. Merci à F. Carnesecchi, O. Leisibach, R. Köchli, P. Hengartner et P. Appelfelder pour leur assistance sur le terrain et au laboratoire. Nous remercions également D. Schmatz pour les données climatiques interpolées et B. Eilmann, P. Weber et H. Bugmann pour les discussions constructives. Ce travail fût soutenu financièrement par le Secrétariat d'Etat à la formation, à la recherche et à l'innovation sous l'action FP0703 et par le Programme de recherche OFEV/WSL «Forêts et changement climatique».

\section{Références}

BECKER M, BERT G, LANDMANN G, LEVY G, RAMEAU J ET AL (1995) Growth and decline symptoms of silver fir and norway spruce in Northeastern France: relation to climate, nutrition and silviculture. In: Landmann G, Bonneau M, Kaennel M, editors. Forest decline and atmospheric deposition effects in the French mountains. Berlin: Springer. pp. 120-142.

BIGLER C, BRÄKER O, BUGMANN H, DOBBERTIN M, RIGLING A (2006) Drought as an inciting mortality factor in Scots pine stands of the Valais, Switzerland. Ecosystems 9: 330-343.

BODENKUNDE AG (2005) Bodenkundliche Kartieranleitung. Stuttgart: Schweizerbart. 438 p.

BREDA N, HUC R, GRANIER A, DREYER E (2006) Temperate forest trees and stands under severe drought: a review of ecophysiological responses, adaptation processes and long-term consequences. Ann For Sci 63: 625-644.

C2SM (2011) Swiss climate change scenarios CH2011. Zurich: Eidg Techn Hochschule, Center Climate Systems Modeling. 88 p.

DESPLANQUE C, ROLLAND C, SCHWEINGRUBER FH (1999) Influence of species and abiotic factors on extreme tree ring modulation: Picea abies and Abies alba in Tarentaise and Maurienne (French Alps). Trees-Struct Funct 13: 218-227.

EILMANN B, BUCHMANN N, SIEGWOLF R, SAURER M, CHERUBINI P ET AL (2010) Fast response of Scots pine to improved water availability reflected in tree-ring width and $013 \mathrm{C}$. Plant Cell Environm 33: 1351-1360.

EILMANN B, ZWEIFEL R, BUCHMANN N, GRAF PANNATIER E, RIGLING A (2011) Drought alters timing, quantity, and quality of wood formation in Scots pine. J Experim Bot 62: 2763-2771.

EILMANN B, RIGLING A (2012) Tree-growth analyses to estimate tree species' drought tolerance. Tree Physiol 32: 178-187.

FRITTS HC (1976) Tree rings and climate. London: Academic Press. $567 \mathrm{p}$.

HENTSCHEL R ET AL (2014) Norway spruce physiological and anatomical predisposition to dieback. For Ecol Manage 322: 27-36.

JANSEN K, SOHRT J, KOHNLE U, ENSMINGER I, GESSLER A (2013) Tree ring isotopic composition, radial increment and height growth reveal provenance-specific reactions of Douglas-fir towards environmental parameters. Trees-Struct Funct 27: 37-52. 
LASSOIE JP, SALO DJ (1981) Physiological response of large Douglas-fir to natural and induced soil-water deficits. Can J For Res 11: 139-144.

LEBOURGEOIS F, LEVY G, AUSSENAC G, CLERC B, WILLM F (1998) Influence of soil drying on leaf water potential, photosynthesis, stomatal conductance and growth in two black pine varieties. Ann For Sci 55: 287-299.

LEBOURGEOIS F, RATHGEBER CBK, ULRICH E (2010) Sensitivity of French temperate coniferous forests to climate variability and extreme events (Abies alba, Picea abies and Pinus sylvestris). J Veg Sci 21: 364-376.

LEO M ET AL (2014) Evaluating the effect of plant water availability on inner alpine coniferous trees based on sap flow measurements. Eur J For Res 133: 691-698.

LEVESQUE M (2013) Drought response of five conifers along an ecological gradient in Central Europe: A multiproxy dendroecological analysis. Zurich: Eidg Techn Hochschule, PhD Thesis 21453. $125 \mathrm{p}$.

LEVESQUE $M$, SAURER $M$, SIEGWOLF R, EILMANN B, BRANG P ET AL (2013) Drought response of five conifer species under contrasting water availability suggests high vulnerability of Norway spruce and European larch. Glob Chang Biol 19: 3184-3199.

LEVESQUE M, RIGLING A, BUGMANN H, WEBER P, BRANG P (2014A) Growth response of five co-occurring conifers to drought across a wide climatic gradient in Central Europe. Agric For Meteorol 197: 1-12.

LEVESQUE M, SIEGWOLF R, SAURER M, EILMANN B, RIGLING A (2014B) Increased water-use efficiency does not lead to enhanced tree growth under xeric and mesic conditions. New Phytol 203: 94-109.

MCCARROLL D, LOADER NJ (2004) Stable isotopes in tree rings. Quat Sci Rev 23: 771-801.

ORWIG D, ABRAMS M (1997) Variation in radial growth responses to drought among species, site, and canopy strata. TreesStruct Funct 11: 474-484.

PASHO E, CAMARERO J, DE LUIS M, VICENTE-SERRANO S (2011) Impacts of drought at different time scales on forest growth

\section{Reaktion einheimischer und exotischer Koniferen auf Trockenheit: eine dendro- ökologische Untersuchung}

Entlang eines klimatischen Gradienten, der den Alpennordhang, das Mittelland, die Juraeinhänge und die Zentralalpen umfasste, wurde die Trockenheitsempfindlichkeit von drei einheimischen und zwei exotischen Koniferenarten untersucht (Fichte, Waldföhre, Lärche; Douglasie, Schwarzföhre). Insgesamt wurden 770 Bäume von 14 Standorten beprobt. Um die Trockenheitsempfindlichkeit abzuschätzen, wurden dendroökologische Analysen für den Zeitraum 1941-2006 durchgeführt. Während auf den trockenen Standorten der Zentralalpen im Frühling auftretende Wasserdefizite das Wachstum stark reduzierten, waren es auf den mesischen Standorten des Mittellands und der Juraeinhänge sommerliche Trockenperioden. Die Fichte, die Lärche und die Waldföhre waren am anfälligsten auf aussergewöhnliche Trockenheit, was bedeutet, dass ihr Produktionsvermögen und - je nach Standort - ihr Überleben gefährdet sein können, wenn das Klima wärmer und trockener wird. Das Wachstum von Douglasie und Schwarzföhre zeigte eine geringere Sensitivität auf Trockenheit und es war höher als dasjenige der einheimischen Arten auf dem Klimagradienten. Baumarten auszuwählen, die unterschiedliche, komplementäre Strategien hinsichtlich Trockenheit aufweisen, könnte die Produktivität und die Wassernutzungseffizienz von Waldbeständen sichern und so deren Resistenz gegenüber dem Klimawandel erhöhen. across a wide climatic gradient in north-eastern Spain Agric For Meteorol 151: 1800-1811.

SCHMID I, KAZDA M (2002) Root distribution of Norway spruce in monospecific and mixed stands on different soils. For Ecol Manage 159: 37-47.

SERGENT AS, ROZENBERG P, BREDA N (2014) Douglas-fir is vulnerable to exceptional and recurrent drought episodes and recovers less well on less fertile sites. Ann For Sci 71: 697-708.

THORNTHWAITE C (1948) An approach toward a rational classification of climate. Geogr Rev 38: 55-94.

THORNTON PE, RUNNING SW, WHITE MA (1997) Generating surfaces of daily meteorological variables over large regions of complex terrain. J Hydrol 190: 214-251.

VAN DER MAATEN-THEUNISSEN M, KAHLE HP, VAN DER MAATEN E (2013) Drought sensitivity of Norway spruce is higher than that of silver fir along an altitudinal gradient in southwestern Germany. Ann For Sci 70: 185-193.

WALTHERT L, GRAF PANNATIER E, MEIER ES (2013) Shortage of nutrients and excess of toxic elements in soils limit the distribution of soil-sensitive tree species in temperate forests. For Ecol Manage 297: 94-107.

WEBER P, BUGMANN H, RIGLING A (2007) Radial growth responses to drought of Pinus sylvestris and Quercus pubescens in an inner-Alpine dry valley. J Veg Sci 18: 777-792.

WELLS N, GODDARD S, HAYES M (2004) A self-calibrating Palmer drought severity index. J Clim 17: 2335-2351.

WERMELINGER B (2004) Ecology and management of the spruce bark beetle Ips typographus - a review of recent research. For Ecol Manage 202: 67-82.

ZANG C (2011) Growth reactions of temperate forest trees to summer drought - a multispecies tree-ring network approach. Munich: Techn Univ München, Chair Ecoclimatology, PhD Thesis. $137 \mathrm{p}$.

ZANG C, PRETZSCH H, ROTHE A (2012) Size-dependent responses to summer drought in Scots pine, Norway spruce and common oak. Trees-Struct Funct 26: 557-569.

\section{Drought response of native and non-native conifers: a dendroecological study}

The sensitivity to drought of five conifers was studied along a climatic gradient covering the northern flank of the Swiss Alps, the Swiss Plateau, the Jura foothills and the central Alps. Three native species, i.e., Norway spruce, Scots pine and European larch, and two non-native species, i.e., Douglas fir and black pine, were analyzed. A total of 770 trees on 14 sites were sampled. To assess the sensitivity of conifers to drought, dendroecological analyses were performed for the period 1941-2006. On dry sites in the central Alps, spring water deficits sharply reduce growth, while on mesic sites located on the Swiss Plateau and the Jura foothills summer droughts significantly restrict growth. Spruce, larch and Scots pine are, depending on the site conditions, the most vulnerable species to extreme droughts, which implies that their long-term performance and survival may be compromised if the climate becomes warmer and drier. Conversely, black pine and Douglas fir were less drought-sensitive and grew faster than native species along the gradient. Selecting species with different but complementary strategies regarding drought could secure the productivity and efficiency of water use of forest stands and increase their resistance to climate change. 